\title{
EFFECTS OF UNIT-CELL BOUNDARY TYPE ON THE ELECTROMECHANICAL PROPERTIES OF RANDOMLY DISTRIBUTED MULTIFUNCTIONAL COMPOSITE STRUCTURES
}

\author{
Joshua Martin, Krishna Challagulla \\ Bharti School of Engineering, Laurentian University \\ Sudbury, Canada \\ Email: krishna.challagulla@laurentian.ca
}

\begin{abstract}
The unit-cell composition of three-dimensional finite element models for 3-0 and 3-1 type polymer (PVDF) ceramic (BaTiO3) and ceramic (PZT-7A) - ceramic (BaTiO3) structures are compared to determine the effects of fiber interaction at the surface of the unit-cell on the effective elastic, piezoelectric and dielectric properties of the multifunctional composite systems. The first unit-cell type examined has enclosed fibers that are completely contained within its boundaries, the second type has fibers that are contained within the sides of the unit-cell but can be cut at the top and bottom surfaces, and the third type has fibers that can be cut on the top, bottom and side surfaces of the unit-cell. All cut fibers are matched on opposing surfaces for continuity. Randomly distributed and aligned circular fibers, randomly distributed and randomly oriented circular fibers, and one central enclosed fiber with varying volume fractions and aspect ratios are compared with these three unit-cell structures. Results show that fiber models display greater or equal values of $\mathrm{C}_{22}$ when compared to aligned or randomly oriented fibers for all cases except aspect ratio 1 polymer-ceramic structures. The third type of unit-cell shows the highest $\mathrm{e}_{22}$ values for single, aligned and randomly oriented fiber structures, except for the aspect ratio 10 polymerceramic case where the second type of unit-cell has greater results for aligned and single fibers. Finally, it can generally be seen that randomly oriented fibers have smaller values than similar aligned and single fiber structures with the exception being $\mathrm{C}_{22}$ of the ceramic-ceramic structures.
\end{abstract}

Keywords-component; Multifunctional composites, finite element analysis, piezoelectricity, random fibers, smart structures

\section{INTRODUCTION}

Piezoelectric materials are being used more commonly for commercial and industrial applications due to their electromechanical properties. In products such as sensors, actuators and hydrophones their unique behavior makes them useful even as monolithic materials. Their properties, however, can be enhanced by combining multiple of these materials in a multifunctional composite structure. These composite systems can include many different types of materials, though the ones being examined in this study will be of the polymer-ceramic and ceramic-ceramic type.
Structured piezoelectric multifunctional composites have been studied fairly extensively from an analytical, numerical and experimental perspective. Kar-Gupta and Venkatesh determined the effects of geometry and grain-size on 3-0, 3-1 and 3-3 fibrous structures in [1]. Wu et al. examine the actuation performance of barium titanate nanoparticles in an epoxy resin in [2]. Yavarow and Erturk [3] develop a nonlinear elastodynamic model for fibrous piezoelectric composites, validating their mathematical model against experimental results.

This being said, randomly distributed and randomly oriented multifunctional composite structures have not been nearly as established. There has been some research done such as Berger et al. in [4], however a complete understanding of the effects of different structures on the effective electromechanical properties of random fiber multifunctional composites is not currently available. This study will try and determine how the interaction between fibers and the surface of a unit-cell will affect the overall properties. Thus, the objectives are:

(i) To develop a unit-cell based finite element model that will predict the elastic, dielectric and piezoelectric properties of several 3-0 and 3-1 type multifunctional composites with different boundary types at varying aspect ratios, volume fractions and cross-sectional shapes for aligned and randomly oriented fibers.

(ii) To systematically characterize the effects of unit-cell boundary structure on the effective electromechanical properties of multifunctional composites.

\section{PiEzoelectric MATERIAls}

A total of three piezoelectric materials are used in this study: barium titanate $\left(\mathrm{BaTiO}_{3}\right)$, polyvinylidene fluoride (PVDF) and lead-zirconate titanate (PZT-7A). Their properties are outlined in table 1 on the next page. PVDF is used as the matrix polymer in the polymer-ceramic models, while PZT-7A is the matrix ceramic in the ceramic-ceramic case. $\mathrm{BaTiO}_{3}$ acts as the fiber in all composite structures. For this study, the matrix was poled in the 2-direction while the fibers were poled along their longitudinal axis for maximum effect, this is recognized as being an ideal case. 


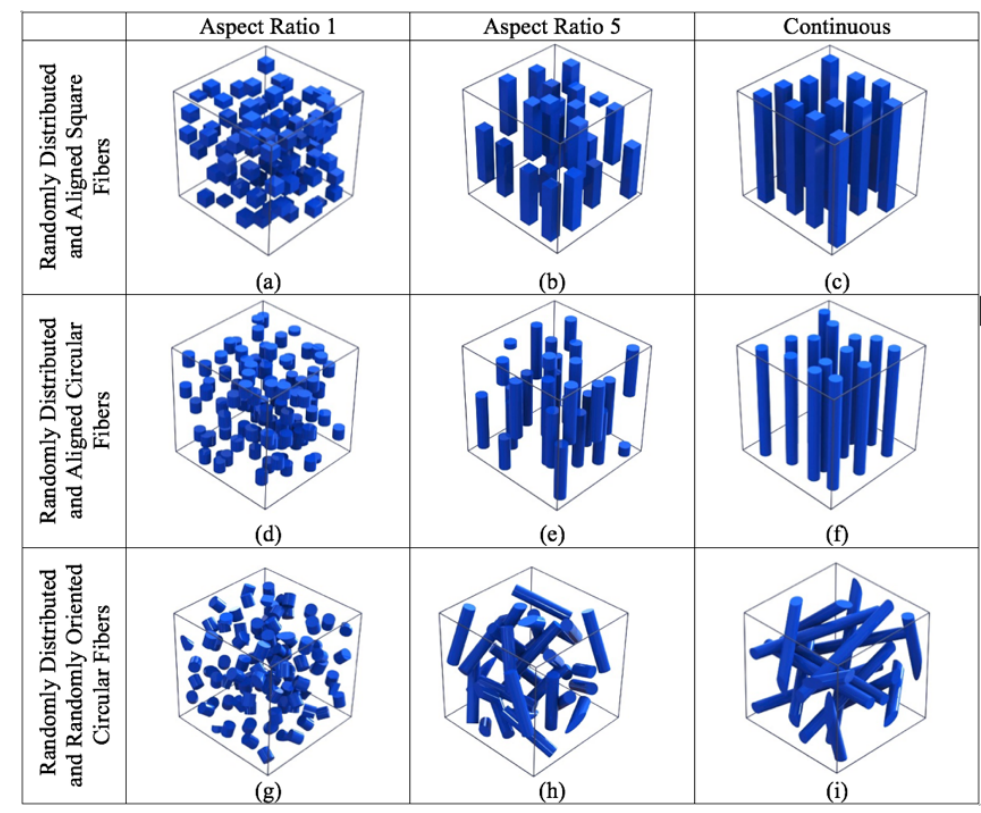

Figure 1: Schematic illustrating nine multifunctional composite structures based on fiber aspect ratio, cross-sectional shape, distribution and orientation.

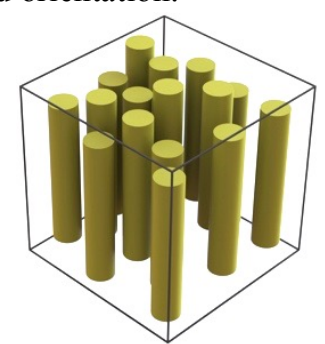

(a)

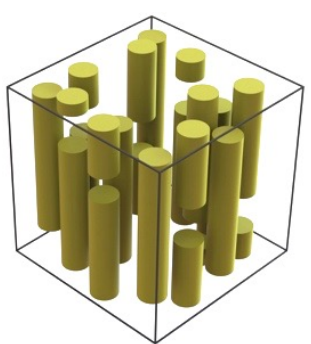

(b)

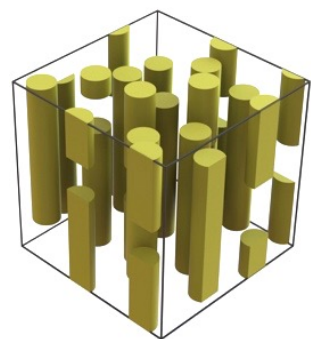

(c)

Figure 2: Unit-cell boundary types: (a) type 1 - fibers completely enclosed; (b) type 2 - fibers enclosed within the sides and not the top; (c) type 3 - fibers not enclosed within the surfaces.

TABLE I. MATERIAL PROPERTIES OF $\mathrm{BATIO}_{3}$, PZT-7A AND PVDF

\begin{tabular}{ccccc}
\hline Material Properties & & $\mathrm{BaTiO}_{3}$ & PZT-7A & PVDF \\
\hline $\mathrm{C}_{11}^{\mathrm{E}}=\mathrm{C}_{33}^{\mathrm{E}}$ & {$[\mathrm{GPa}]$} & 150.40 & 148.00 & 4.840 \\
$\mathrm{C}_{12}^{\mathrm{E}}=\mathrm{C}_{23}^{\mathrm{E}}$ & {$[\mathrm{GPa}]$} & 65.94 & 74.20 & 2.220 \\
$\mathrm{C}_{13}^{\mathrm{E}}$ & {$[\mathrm{GPa}]$} & 65.63 & 76.20 & 2.720 \\
$\mathrm{C}_{22}^{\mathrm{E}}$ & {$[\mathrm{GPa}]$} & 145.50 & 131.00 & 4.630 \\
$\mathrm{C}_{44}^{\mathrm{E}}=\mathrm{C}_{66}^{\mathrm{E}}$ & {$[\mathrm{GPa}]$} & 43.86 & 25.40 & 0.052 \\
$\mathrm{C}_{55}^{\mathrm{E}}$ & {$[\mathrm{GPa}]$} & 42.37 & 35.90 & 1.060 \\
$\mathrm{~K}_{11}=\kappa_{33}$ & {$[\mathrm{nC} / \mathrm{Vm}]$} & 12.8 & 3.984 & 0.0664 \\
$\mathrm{\kappa}_{22}$ & {$[\mathrm{nC} / \mathrm{Vm}]$} & 15.1 & 2.081 & 0.0708 \\
$\mathrm{e}_{16}=\mathrm{e}_{34}$ & {$\left[\mathrm{C} / \mathrm{m}^{2}\right]$} & 11.40 & 9.31 & -0.001999 \\
$\mathrm{e}_{21}=\mathrm{e}_{23}$ & {$\left[\mathrm{C} / \mathrm{m}^{2}\right]$} & -4.32 & -2.324 & 0.004344 \\
$\mathrm{e}_{22}$ & {$\left[\mathrm{C} / \mathrm{m}^{2}\right]$} & 17.36 & 10.9 & 0.1099 \\
\hline & & & & \\
\hline
\end{tabular}

Piezoelectric materials are governed and fully defined by 21 elastic, 18 piezoelectric and 6 permittivity constants [5]. In the first equation, $\sigma$ represents a second order stress tensor, $C^{E}$ a fourth order elasticity tensor (at zero electric field), $\varepsilon$ a second order strain tensor, $\mathrm{E}$ an electric field vector, D an electric displacement vector, e a third order coupling tensor and $\kappa^{\varepsilon}$ a second order permittivity tensor (at zero strain). The indices $\mathrm{i}, \mathrm{j}, \mathrm{k}$ and $\mathrm{l}$ are remapped in the following way: $11 \rightarrow$ $1,22 \rightarrow 2,33 \rightarrow 3,23 \rightarrow 4,13 \rightarrow 5$ and $12 \rightarrow 6$.

$$
\begin{gathered}
\sigma_{i j}=C_{i j k l}^{E} \varepsilon_{k l}-e_{i j k} E_{k} \\
D_{i}=e_{i k l} \varepsilon_{k l}+\kappa_{i j}^{\varepsilon} E_{j}
\end{gathered}
$$

\section{Composite Unit-Cell Structure}

The multifunctional composite structures analyzed in this study were generated using python scripts which were then run using the commercially available finite element software, ABAQUS. The code developed created fibers with specified cross-sectional shapes and aspect ratios, then placed them randomly in a non-intersecting fashion within a unit-cell until a desired volume fraction was reached. Fibers were also constrained in relation to the boundary of the unit-cell in three ways, as shown in figure 2 :

- Type 1: fibers are completely enclosed within the boundary of the unit-cell. No fiber gets cut at or even touches any surface of the unit-cell.

- Type 2: fibers are enclosed within the four sides of the unit cell but can be cut on the top and bottom surfaces.

- Type 3: fibers can be cut on any surface of the unit-cell. 
All fibers cut on one surface have a complementary fiber on the opposing surface to have continuity if multiple unit-cells were to be attached, shown below in figure 3 .

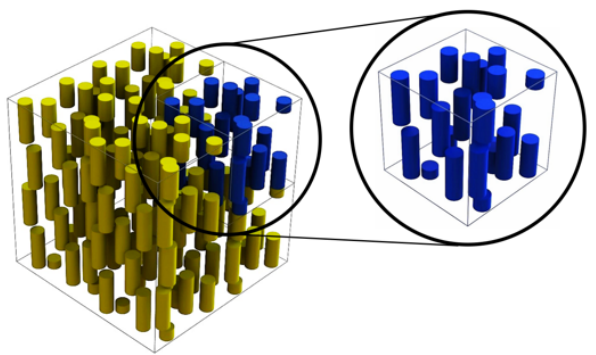

Figure 3: Continuity at unit-cell boundaries using complementary fibers.

The finite element models were run using threedimensional four-node linear piezoelectric tetrahedron elements (C3D4E). Nodes were given four degrees of freedom, each of the three directions and one electric potential (1, 2, 3 and 9, respectively). Fiber diameter and length was also kept constant in order to maintain aspect ratio. The number of fibers within a unit-cell could have been increased by keeping aspect ratio constant and reducing the diameter, however this was not done in this study to keep fibers consistent, which limited the volume fraction to approximately $25 \%$ [6].

\section{RESUlTS}

Finite element methods were used to analyze the various composite structures described in section 3 in order to determine effective elastic, dielectric and piezoelectric

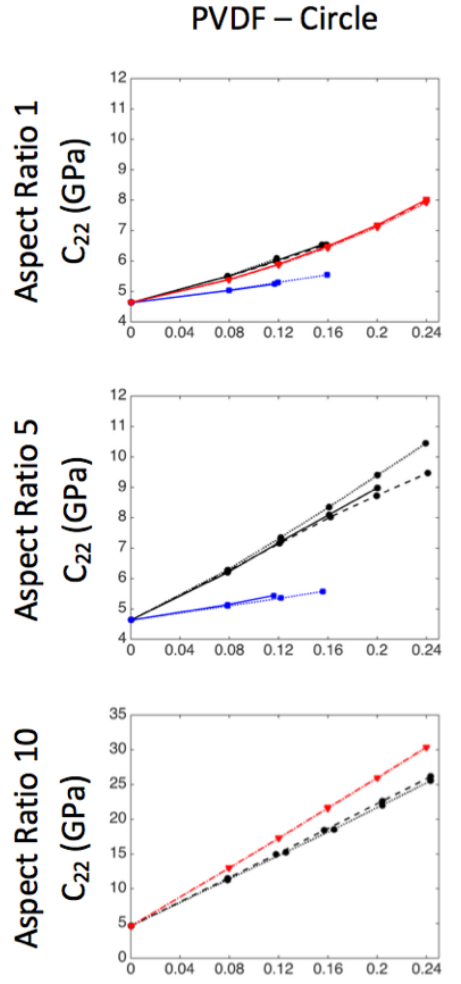

(a)
PVDF - Square
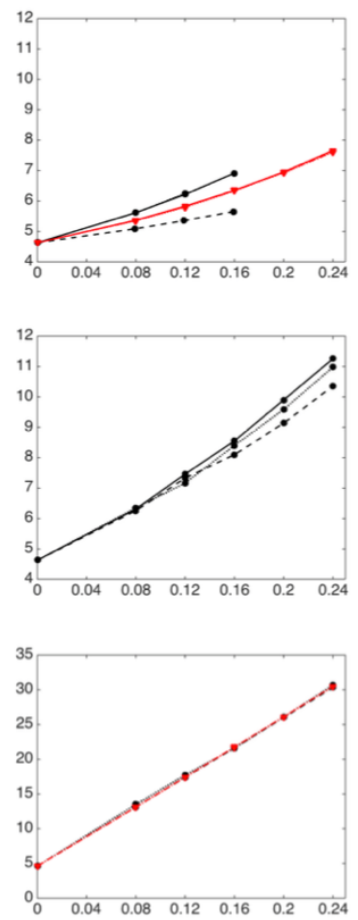

(b)

properties. The results plotted in the graphs of figure 4 show these effective properties for each structure at a given aspect ratio, volume fraction, cross-sectional shape, material and boundary type. Results displayed for any randomly distributed fiber structures are averages calculated from multiple iterations of the model. The cross-sectional fiber shapes examined are circular and square, while the material compositions studied are polymer-ceramic and ceramic-ceramic. Aspect ratio 1, 5 and 10 (short, medium and long fibers, respectively) are also compared with the three types of boundary structures.

The random fiber results from this study are initially compared to single fiber results with comparable structures to determine their accuracy. The circular single fiber results were verified against Kar-Gupta and Venkatesh [1], as well as Bowen et al [7], and Brito-Santana [8]. Single fiber results with the first type of boundary structure are shown to have nearly identical values and trends as those in [1,7 and 8], which is as expected since the structures are the same. There is some variance with different boundary structures and with randomly distributed fibers, however the models are overall in good agreement.

Square fibers were also compared with Kar Gupta and Venkatesh [9]. The aspect ratio 10 randomly distributed and single fiber structures were compared with those of [9] and showed similar trends. Though both studies examined polymerceramic and ceramic-ceramic structures with the same materials, the combinations of materials were different so an exact verification could not be made.

PZT - Circle
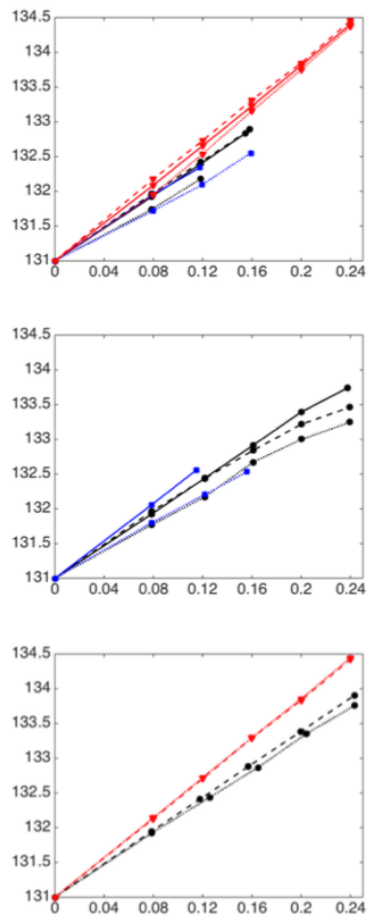

(c)
PZT - Square
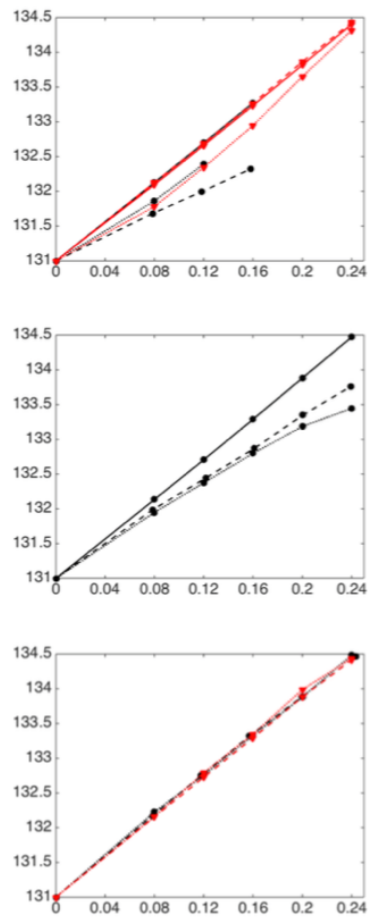

(d) 
PVDF - Circle
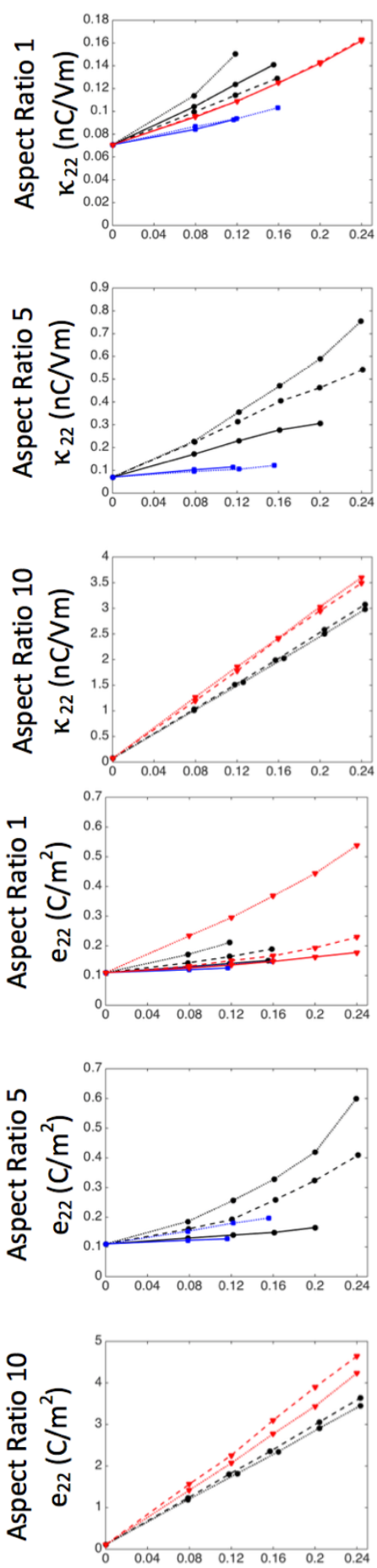

(a)
PVDF - Square
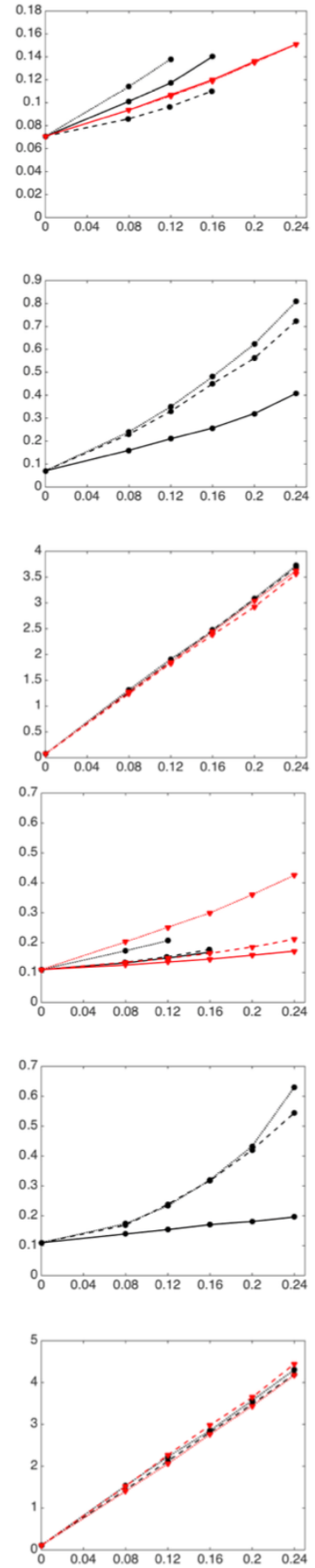

(b)
PZT - Circle
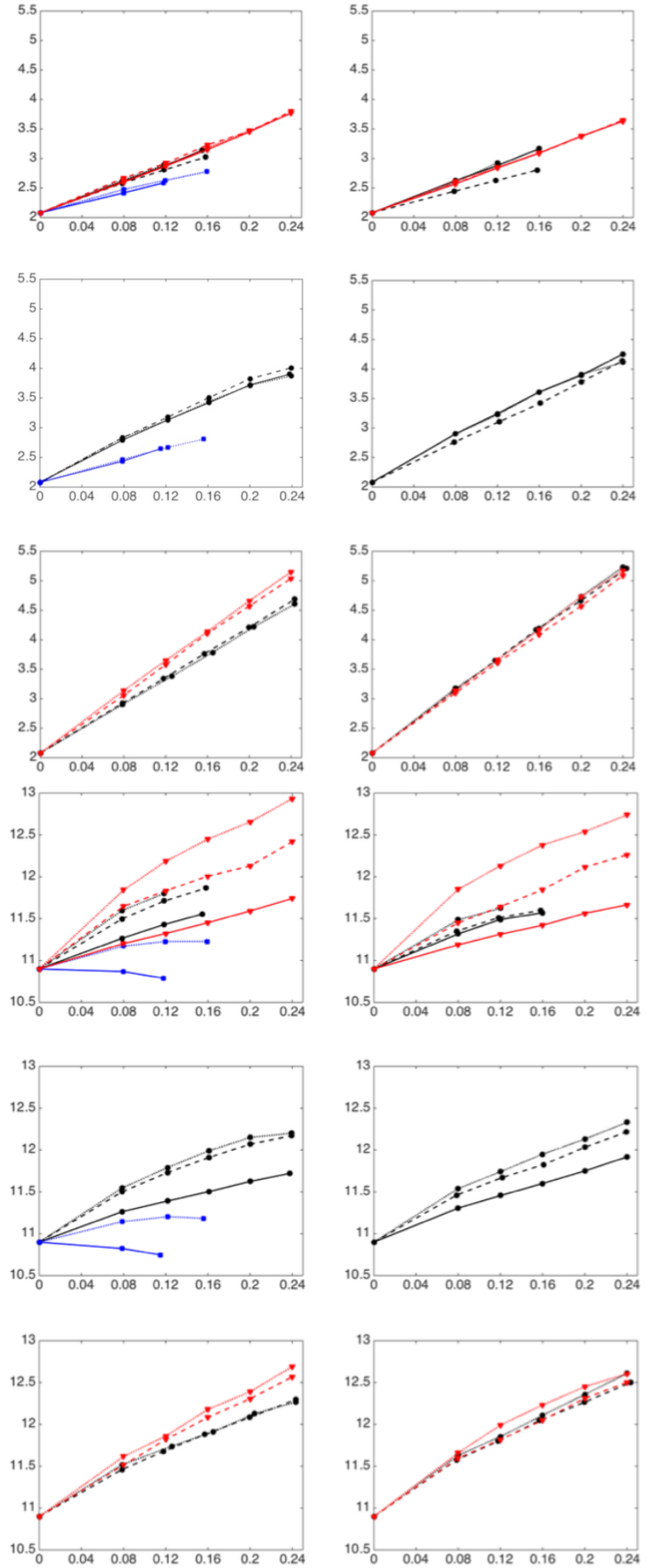

(c)

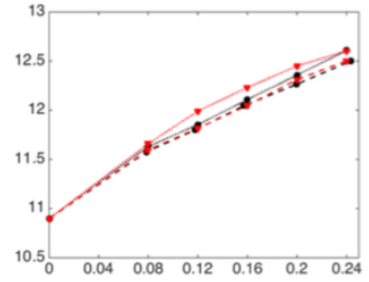

(d)

Figure 4: Variation of effective properties with respect to fiber volume fraction in (a) polymer (PVDF) - ceramic (BaTiO3) system with aligned and randomly distributed circular fibers, randomly oriented and randomly distributed circular fibers, and single aligned fibers (b) polymer-ceramic system with aligned, randomly distributed and single square fibers, (c) ceramic (PZT-7A) - ceramic (BaTiO3) system with aligned and randomly distributed circular fibers, randomly oriented and randomly distributed, and single aligned circular fibers, and (d) ceramic-ceramic system with aligned, randomly distributed and single square fibers - multifunctional composite structures. 
For $\mathrm{C}_{22}$, single fiber models display greater or equal values compared to aligned and randomly oriented fibers in all cases but aspect ratio 1 polymer-ceramic structures. In the longitudinal direction, it appears that the length and continuity of the fiber plays a more significant role in increasing mechanical properties than boundary type. It can, however, also be seen that the first type of boundary structure has the highest $\mathrm{C}_{22}$ value for a given structure when compared to the second and third boundary types with the exception of aspect ratio 1 ceramic-ceramic circular structures and aspect ratio 5 polymerceramic circular structures.

Randomly oriented fibers are generally seen to have the lowest values for $\mathrm{C}_{22}, \mathrm{e}_{22}$ and $\kappa_{22}$ when compared to aligned and single fibers with the exception being $\mathrm{C}_{22}$ of the ceramicceramic structures. Aligned fiber multifunctional composite systems have several distinct advantages when compared to these randomly oriented structures. They can be placed more efficiently in the unit-cell, leading to higher packing factors as well as providing generally flexible structures [10] with low acoustic impedance values [11]. Randomly oriented fiber structures, however, can provide higher values in transverse directions compared to aligned structures and can be easier to manufacture [12-15].

\section{CONCLUSION}

Random fiber multifunctional composites have a wide variety of advantages compared to structured and single fiber structures. Their ease in manufacturing and higher values in transverse directions make them useful and often less expensive. However, as the applications for these composites becomes more pronounced, their complexity will increase and the numerical models used to predict their behaviour will need to be improved. The purpose of this study was specifically to determine how the effective electromechanical properties of multifunctional composites might be affected by changes in the interaction between fibers and the surface of the unit-cell. At given volume fractions of fibers, aspect ratios, materials and cross-sectional shapes, the effects of the boundary structure of a unit-cell were found to be the following:

(i) $\mathrm{C}_{22}$ values were more commonly larger as a result of longer and continuous fibers rather than based on a unitcell boundary type, however the first type of boundary displays generally higher values compared to the second and third type except for aspect ratio 1 ceramic-ceramic circular structures and aspect ratio 5 polymer-ceramic circular structures.

(ii) The highest $\mathrm{e}_{22}$ values for single, aligned and randomly oriented fiber structures except for the aspect ratio 10 polymer-ceramic case was the third type of unit-cell.

(iii) Randomly oriented fibers have the lowest values in general when compared to aligned and single fiber structures, with the exceptions of $\mathrm{C}_{22}$ of the ceramicceramic structures.

This study presents an overview of the effects different fiber-surface interactions can have on the overall properties of a unit-cell. When creating models to study the behaviour of random fiber multifunctional composite structures, the conclusions drawn here can help identify discrepancies in electromechanical properties between models that appear to be the same. As is shown, the same structure with different surface-fiber interactions can have varying effective mechanical, piezoelectric and dielectric properties without having a different core structure. The large variety of structures being compared in this study with varying aspect ratios, volume fractions, materials and cross-sectional shapes provides a baseline for future numerical models with more complex structures and unitcell surfaces.

\section{REFERENCES}

[1] R. Kar-Gupta, T.A. Venkatesh, "Electromechanical response of piezoelectric composites: Effects of geometric connectivity and grain size," Acta Materialia, vol. 56, pp. 3810-3823, 2008.

[2] M. Wu, X. Yuan, H. Luo, H. Chen, K. Zhou, D. Zhang, "Enhanced actuation performance of piezoelectric fiber composites induced by incorporated $\mathrm{BaTiO} 3$ nanoparticles in epoxy resin," Physics Letters A, vol. 381, pp. 1641-1647, 2017.

[3] T. P. Yavarow, A. Erturk, "Nonlinear elastodynamics of piezoelectric macro-fiber composites with interdigitated electrodes for resonant actuation," Composite Structures, vol. 187, pp. 137-143, 2018.

[4] H. Berger, S. Kari, U. Gabbert, R. Rodriguez-Ramos, J. BravoCastillero, R. Guinovart-Diaz, "Calculation of effective coefficients for piezoelectric fiber composites based on a general numerical homogenization technique," Composite Structures, vol. 71, pp. 397400, 2005.***

[5] P.W. Bosse, K.S. Challagulla., T.A. Venkatesh, "Effects of foam shape and porosity aspect ratio on the electromechanical properties of 3-3 piezoelectric foams," Acta Materialia, vol. 60, pp. 6464-6475, 2012.

[6] S. Kari, H. Berger, U. Gabbert, "Numerical evaluation of effective material properties of randomly distributed short cylindrical fibre composites," Computational Materials Science, vol. 39, pp. 198-204, 2007.

[7] R. Kar-Gupta, T.A. Venkatesh, "Electromechanical response of 1-3 piezoelectric composites: A numerical model to assess the effects of fiber distribution," Acta Materialia, vol. 55, pp. 1275-1292, 2007.

[8] X.J. Lin, K.C. Zhou, X.Y. Zhang, D. Zhang, "Development, modeling and application of piezoelectric fiber composites," Transactions of Nonferrous Metals Society of China, vol. 23, pp. 98-107, 2013.

[9] Y. Ismail, Y. Sheng, D. Yang, J. Ye, "Discrete element modelling of unidirectional fibre-reinforced polymers under transverse tension," Composites Part B: Engineering, vol. 73, pp. 118-125, 2015.

[10] M. Chalh, S. Vedraine, B. Lucas, B. Ratier, "Plasmonic Ag nanowire network embedded in zinc oxide nanoparticles for inverted organic solar cells electrode," Solar Energy Materials \& Solar Cells, vol. 152, pp. 34-41, 2016.

[11] S. Kari, H. Berger, R. Rodriguez-Ramos, U. Gabbert, "Computational evaluation of effective material properties of composites reinforced by randomly distributed spherical particles," Composite Structures, vol. 77, pp. 223-231, 2007.

[12] T. Vaughan, C. McCarthy, "A micromechanical study on the effect of intra-ply properties on transverse shear fracture in fibre reinforced composites," Compos A Appl Sci Manuf, vol. 42, pp. 1217-28, 2011.

[13] T. Vaughan, C. McCarthy, "Micromechanical modelling of the transverse damage behaviour in fibre reinforced composites," Compos Sci Technol, 71, pp. 388-96, 2011

[14] C.R. Bowen, A. Perry, H. Kara, S.W. Mahon, "Analytical modelling of 3-3 piezoelectric composites," Journal of European Ceramic Society, 21, pp. 1463-1467, 2001.

[15] H. Brito-Santana, R. de Medeiros, R. Rodriguez-Ramos, V. Tita, "Different interface models for calculating the effective properties in piezoelectric composite materials with imperfect fiber-matrix adhesion," Composite Structures, 151, pp. 70-80, 2016. 